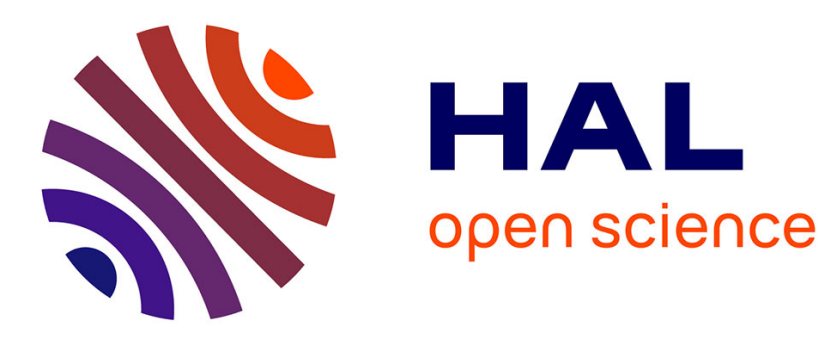

\title{
Distributed Power Allocation Strategy in Shallow Water Acoustic Interference Channels
}

\author{
Antony Pottier, François-Xavier Socheleau, Christophe Laot
}

\section{To cite this version:}

Antony Pottier, François-Xavier Socheleau, Christophe Laot. Distributed Power Allocation Strategy in Shallow Water Acoustic Interference Channels. SPAWC 2016: IEEE 17th International Workshop on Signal Processing advances in Wireless Communications, Jul 2016, Edinburgh, United Kingdom. pp.1 - 5, 10.1109/SPAWC.2016.7536793 . hal-01369655

\section{HAL Id: hal-01369655 \\ https://hal.science/hal-01369655}

Submitted on 11 Jun 2021

HAL is a multi-disciplinary open access archive for the deposit and dissemination of scientific research documents, whether they are published or not. The documents may come from teaching and research institutions in France or abroad, or from public or private research centers.
L'archive ouverte pluridisciplinaire HAL, est destinée au dépôt et à la diffusion de documents scientifiques de niveau recherche, publiés ou non, émanant des établissements d'enseignement et de recherche français ou étrangers, des laboratoires publics ou privés. 


\title{
Distributed Power Allocation Strategy in Shallow Water Acoustic Interference Channels
}

\author{
Antony Pottier, Francois-Xavier Socheleau, Christophe Laot \\ Institut Mines-Telecom; TELECOM Bretagne, UMR CNRS 6285 Lab-STICC \\ Email: \{antony.pottier,fx.socheleau,christophe.laot\}@telecom-bretagne.eu
}

\begin{abstract}
Due to the absence of spectrum regulation and limited bandwidth, underwater acoustic (UA) systems are prone to interfere with each other. In this paper, a decentralized power allocation strategy is proposed for multiple OFDM UA links sharing the same physical resource. These links are supposed to be noncooperative and aim at selfishly maximize their own information rate. Each link is assumed to only know the statistics of its channel and the overall noise plus interference power spectral density. A game-theoretic formulation, which explicitly takes into account the random time-varying nature of the underwater acoustic channel as well as the low speed of sound, is derived. Numerical simulations show the strong benefit of proposed approach in highly interfering channels.
\end{abstract}

Index Terms-Underwater acoustic communications, power allocation, OFDM, interference channel, Nash equilibrium.

\section{INTRODUCTION}

$\mathbf{U}$ NLIKE the radio-communication spectrum, the use of the underwater acoustic (UA) spectrum is not restricted by regulatory bodies [1]. Consequently, communication systems are not only constrained by a complex propagation medium [2] but also by interferences potentially caused by other entities wishing to use the same resource.

To improve the coexistence of heterogeneous sources underwater, its is not conceivable to apply a fixed assignment that restricts the access of a particular bandwidth for a specific kind of system/application since the total bandwidth is very limited. A more suitable strategy is to bring some intelligence to communicating devices that will allow them to be aware of their environment and automatically adapt their transmission parameters to the context in which they operate. This approach is much more flexible as it is a decentralized way to deal with noncooperative UA devices competing for the same resource.

Recent results have shown that adaptive modulation can be truly beneficial for single user UA communication links [3], [4]. In this work, we ambition to demonstrate that adaptation is also relevant for the multiuser case. More precisely, we seek to find a distributed power allocation strategy across frequencies for a set of competitive UA systems that try communicate in the same bandwidth at the same time. In the absence of spectrum regulation and UA communication standards, this scenario is likely to happen underwater. To the best of our knowledge, most of the research efforts on resource allocation for UA multiusers have only focused on homogeneous systems evolving within the same network [5].

The competitive systems considered here are assumed to be fully noncooperative. It means that they are selfish and that they cannot communicate with each other to agree on a fair resource sharing scheme. However, to find reasonable operating points - and not to be in situations where all systems interfere so much that none of them is actually reliable it is necessary that each transmitter optimizes its parameters according to the ambient soundscape. Such an optimization is made possible only if each receiver sends some metrics on the link quality back to its transmitter. A standard framework to design distributed multiobjective optimization algorithms is game theory. Every UA link is a player that competes against the others by choosing a frequency power allocation that maximizes its own performance metric, also called utility function within game theory. The metric chosen in this work is the information rate and we consider UA links in a shallow water channel using OFDM modulation, which is well suited to spectrum sharing problems.

Noncooperative power allocation games over frequency selective interference channels have been widely studied for terrestrial communications [6]-[8]. In these works, distributed maximization of information rate is solved according to the Nash Equilibrium concept. This equilibrium is an outcome of the game where, given the strategies of its rivals, every link is playing its optimal strategy in the sense that any other choice would result in a rate loss. This yields to a solution where all the links adopt a waterfilling strategy against each others. In the cited papers, the system models and resulting game-theoretic formulations often rely on the assumption of known channel realizations. This assumption seems unrealistic in our context because the low speed of sound underwater $\left(\approx 1500 \mathrm{~m} . \mathrm{s}^{-1}\right)$ combined with the rapidly time-varying nature of the medium leads to outdated channel state information (CSI) if short period feedback policies are used.

In this paper, we take these constraints into account. A randomly time-varying, frequency-selective UA channel is considered where the subchannel gains are modeled as Rician processes. The transmitters update their power spectral density (PSD) according to long term statistics on their direct channel and on the overall noise plus interference computed by their respective receivers. Thus, a long period feedback link can be implemented.

The paper is organized as follows. The information rate maximization problem via distributed power allocation is formulated as a game in Section II. In Section III, a solution is proposed using the concept of Nash Equilibrium. We conclude by numerical simulations in Section IV showing that our 
approach can be relevant for multiuser UA communications.

Notations: Uppercase and lowercase boldface letters, e.g. A, x, denote matrices and vectors, respectively. The superscript ${ }^{T}$ denotes transposition. The Hadamard product is denoted by $\odot . \mathcal{L}^{2}$ denotes the vector space of random variables with finite second order moment. Finally, $\mathbb{E}\{$.$\} denotes$ expectation.

\section{PROBLEM FORMULATION}

\section{A. Channel and system model}

We consider an UA interference channel in which $M$ transmitter-receiver links are competing to communicate in the same band. The channel is supposed to be frequencyselective and randomly time-varying. To cope with frequencyselectivity, OFDM signalling with $N$ subcarriers is used for each link. The total bandwidth is $B=N \Delta f$, with $\Delta f$ the subcarrier spacing chosen smaller than the channel coherence bandwidth. As we focus on noncooperative distributed policies, we assume, as in [7], that the interference of other users is treated as additive colored noise at each receiver.

Let $\mathbf{x}_{i}(n)$ be a vector of $L$ zero-mean i.i.d. complex input symbols sent on subchannel $n$ by transmitter $i$. The symbols transmitted by any other user $j \neq i$ are assumed independent from those transmitted by $i$. Transmitters are subject to the following power constraint

$$
\sum_{n=1}^{N} p_{i}(n) \leq P_{i}^{\max }
$$

where $p_{i}(n)$ is the power allocated by transmitter $i$ on the $n^{\text {th }}$ subcarrier.

After cyclic prefix removal and discrete Fourier transform, the channel output observed over $L$ OFDM symbols by the $i^{t h}$ receiver on the $n^{t h}$ subcarrier can be expressed as: ${ }^{1}$

$$
\mathbf{y}_{i}(n)=\mathbf{h}_{i i}(n) \odot \mathbf{x}_{i}(n)+\sum_{j \neq i} \mathbf{h}_{j i}(n) \odot \mathbf{x}_{j}(n)+\mathbf{w}_{i}(n),
$$

where $\mathbf{w}_{i}(n) \sim \mathcal{C N}\left(0, \sigma_{w_{i}}^{2}(n) \mathbf{I}_{L}\right)$ is a Gaussian noise independent of both $\mathbf{x}_{i}(n)$ and $\mathbf{x}_{j \neq i}(n) . \mathbf{h}_{i i}(n) \in \mathbb{C}^{L}$ are the coefficients of the direct subchannel $n, \mathbf{h}_{j i}(n) \in \mathbb{C}^{L}$ are those of the interference subchannel $n$ between transmitter $j$ and receiver $i$. In agreement with [10], [11], the shallow water channel fading process is modeled by a Rician distribution so that, for each OFDM symbol $l, h_{i i}^{(l)}(n) \sim \mathcal{C N}\left(\mu_{i i}(n), \sigma_{i i}^{2}(n)\right)$. The channel statistics are assumed to be constant over a block of $L$ symbols. $^{2}$

\footnotetext{
${ }^{1}$ Doppler effects are supposed to be compensated, and intercarrier interference (ICI) caused by temporal misalignment of received symbols from other transmitters are not explicitly taken into account in our work. These assumptions are made in light of [9], where it is shown that considering ICI in the context of distributed power allocation does not significantly change the problem formulation.

${ }^{2}$ Note that this assumption of block-stationarity is an approximation since, in practice, the taps mean $\mu_{i i}(n)$ can be slowly time-varying as discussed in [11], [12]
}

\section{B. Problem formulation as a noncooperative game}

In this section, we formulate our problem as a strategicform noncooperative game in which the $M$ transmitter-receiver links communicating in the same UA channel are the players of the game. Their strategies are the possible power allocations satisfying constraint (1). Each player competes rationally and seeks to maximize a metric that describes its information rate. Because of the low propagation speed of acoustic waves, the small-scale fast-varying part of the channel does not admit feedback. Therefore, the performance metric can only be related to some "average" information rate. Assuming that the duration corresponding to $L$ OFDM symbols is much greater than the channel coherence time, a standard figure of merit would be

$$
r_{i}(\mathbf{p})=\sum_{n=1}^{N} \mathbb{E}\left\{\log \left(1+\operatorname{SINR}_{i}(n)\right)\right\},
$$

where $\operatorname{SINR}_{i}(n)$ is the signal-to-interference plus noise ratio of link $i$ on subcarrier $n$ :

$$
\operatorname{SINR}_{i}(n)=\frac{\left|h_{i i}(n)\right|^{2} p_{i}(n)}{\sigma_{w_{i}}^{2}(n)+\sum_{j \neq i}\left|h_{j i}(n)\right|^{2} p_{j}(n)},
$$

with $\mathbf{p}=\left[\mathbf{p}_{1}, \cdots, \mathbf{p}_{M}\right]$ and $\mathbf{p}_{i}=\left[p_{i}(1), \cdots, p_{i}(N)\right]^{T}$.

Optimizing the spectral allocation using (3) would require for each receiver $i$ to estimate both its direct channel coefficients and those of the interference channels. While each $h_{i i}^{(l)}(n)$ can be estimated using pilot symbols, the interference cannot be differentiated from noise if we consider a noncooperative context. Therefore, power allocation is reformulated as a robust problem where the optimization is made by considering the worst possible interference. Let $f_{h_{j i}}$ denote the probability density function of $h_{j i}$, the performance metric for the worst case optimization problem is computed as follows

$$
u_{i}(\mathbf{p})=\min _{f_{h_{j i}, i \neq j}: h_{j i} \in \mathcal{L}^{2}} r_{i}(\mathbf{p})
$$

$$
\begin{aligned}
& \stackrel{(a)}{=} \sum_{n=1}^{N} \mathbb{E}\left\{\log \left(1+\frac{\left|h_{i i}(n)\right|^{2} p_{i}(n)}{\sigma_{w_{i}}^{2}(n)+\sum_{j \neq i} \mathbb{E}\left\{\left|h_{j i}(n)\right|^{2}\right\} p_{j}(n)}\right)\right\} \\
& \stackrel{(b)}{\approx} \sum_{n=1}^{N} \log \left(1+\frac{g_{i}(n) \mathbb{E}\left\{\left|h_{i i}(n)\right|^{2}\right\} p_{i}(n)}{\sigma_{w_{i}}^{2}(n)+\sum_{j \neq i} \mathbb{E}\left\{\left|h_{j i}(n)\right|^{2}\right\} p_{j}(n)}\right),
\end{aligned}
$$

where

$$
g_{i}(n)=\frac{K_{i}(n)}{K_{i}(n)+1} e^{-\operatorname{Ei}\left(-K_{i}(n)\right)} .
$$

$K_{i}(n)=\left|\mu_{i i}(n)\right|^{2} /\left|\sigma_{i i}(n)\right|^{2}$ is the Rice factor of subchannel $h_{i i}(n)$ and $\operatorname{Ei}(-x)$ denotes the exponential integral function defined, for $x>0$, as

$$
\operatorname{Ei}(-x)=-\int_{x}^{+\infty} \frac{e^{-t}}{t} d t
$$

In (5), $(a)$ follows from Jensen's inequality for conditional expectations and equality is achieved for Gaussian interferences. Note that in this expression, the denominator only depends on the average power of "noise plus interference" so that the interference needs not to be differentiated from 
noise when estimated by each receiver. Approximation $(b)$ is actually a lower bound of (5)-(a) obtained by adapting the approach described in [13] to Rician channels. For shallow water channels, $K_{i}(n)$ is usually on the order of several decibels [11], [14]. In this context (5)-(b) turns out to be a very tight approximation of (5)- $(a)$.

Compared to most of the literature on game theory applied to power allocation in interference channels [6]-[9], [15], $u_{i}(\mathbf{p})$ explicitly takes the randomly time-varying nature of the channel into account. It can be derived from channel statistics computed by integrating several channel and noise plus interference PSD estimates. Using long-term statistics on the channels can reduce the feedback activity of the receivers and cope with issues related to outdated CSI caused by long feedback delays that are typical in UA channels.

The game can now be formalized as

$$
\mathcal{G}=\left\{\mathcal{M},\left\{\mathcal{P}_{i}\right\}_{i=1}^{M},\left\{u_{i}\right\}_{i=1}^{M}\right\},
$$

where the set of players $\mathcal{M}=\{1, \cdots, M\}$ are the active links in the UA channel, $\mathcal{P}_{i}$ is the strategies space of the player $i \in \mathcal{M}$ defined as the possible power allocations, i.e.

$$
\mathcal{P}_{i}=\left\{\mathbf{p}_{i} \in \mathbb{R}_{+}^{N}: \sum_{n=1}^{N} p_{i}(n) \leq P_{i}^{\max }\right\}
$$

and $u_{i}$ in (5) is the utility function that each player $i$ wants to maximize.

\section{PROPOSED SOLUTION}

\section{A. Best Response and Nash Equilibrium}

Starting from the structure of game $\mathcal{G}$, our purpose is to find for each player $i \in \mathcal{M}$ the optimal strategy $\mathbf{p}_{i}^{\star} \in \mathcal{P}_{i}$ that maximizes its utility function $u_{i}\left(\mathbf{p}_{i}, \mathbf{p}_{-i}^{\star}\right)$, given that other players are also playing their optimal strategies denoted by $\mathbf{p}_{-i}^{\star}=\left[\mathbf{p}_{1}^{\star}, \cdots, \mathbf{p}_{i-1}^{\star}, \mathbf{p}_{i+1}^{\star}, \cdots, \mathbf{p}_{M}^{\star}\right]$. These optimal power allocations are reached non-cooperatively, each link treating the interferences caused by others as noise. Such a strategy profile $\mathbf{p}^{\star}=\left[\mathbf{p}_{1}^{\star}, \cdots, \mathbf{p}_{M}^{\star}\right]$, where no player has an interest to deviate from, is called a Nash Equilibrium (NE) of the game and is formally defined as follows [16]:

Definition 1: A NE of the game $\mathcal{G}$ in (8) is a strategy profile $\mathbf{p}^{\star}$ such that $\forall i \in \mathcal{M}$ and $\forall \mathbf{p}_{i} \in \mathcal{P}_{i}$, we have

$$
u_{i}\left(\mathbf{p}_{i}^{\star}, \mathbf{p}_{-i}^{\star}\right) \geq u_{i}\left(\mathbf{p}_{i}, \mathbf{p}_{-i}^{\star}\right) .
$$

In our context, this definition means that if the UA channel users have allocated their transmit power in such a way that a NE of game (8) is attained, there will be no user which can get a higher mutual information by modifying unilaterally its power allocation. Thus, the solution of our noncooperative power allocation problem will logically be based on the concept of NE.

The existence of a Nash equilibrium for game $\mathcal{G}$ is guaranteed by the Debreu-Glicksberg-Fan theorem [17, Theorem 1.2] which states that there exists at least one (pure-strategy) $\mathrm{NE}$ in every game whose strategic spaces $\mathcal{P}_{i}$ are non-empty compact convex subsets of an Euclidian space and whose utility functions $u_{i}\left(\mathbf{p}_{i}, \mathbf{p}_{-i}\right)$ are continuous in $\left(\mathbf{p}_{i}, \mathbf{p}_{-i}\right)$ and quasi-concave in $\mathbf{p}_{i}$. One can verify that these conditions are satisfied by the strategic spaces defined in (9) and the utility in (5).

Before going further, we need to introduce the concept of best response [16].

Definition 2: The best responses of a player $i$ to a given strategy profile of its opponents $\mathbf{p}_{-i}$ are its strategies $\mathbf{p}_{i}^{\star} \in \mathcal{P}_{i}$ such that

$$
u_{i}\left(\mathbf{p}_{i}^{\star}, \mathbf{p}_{-i}\right) \geq u_{i}\left(\mathbf{p}_{i}, \mathbf{p}_{-i}\right), \forall \mathbf{p}_{i} \in \mathcal{P}_{i} .
$$

Consequently, by combining definitions 1 and 2, we have that a NE is reached when all the players play their best responses simultaneously. To find his best responses, each player aims at solving the following maximization problem

$$
\begin{array}{ll}
\underset{\mathbf{p}_{i}}{\operatorname{maximize}} & u_{i}\left(\mathbf{p}_{i}, \mathbf{p}_{-i}\right) \\
\text { subject to } & \mathbf{p}_{i} \in \mathcal{P}_{i} .
\end{array}
$$

Replacing $u_{i}\left(\mathbf{p}_{i}, \mathbf{p}_{-i}\right)$ by its expression in (5), problem (12) yields the waterfilling solution

$$
p_{i}^{\star}(n)=\left[\frac{1}{\lambda_{i}}-\frac{\sigma_{w_{i}}^{2}(n)+\sum_{j \neq i} \mathbb{E}\left\{\left|h_{j i}(n)\right|^{2}\right\} p_{j}(n)}{g_{i}(n) \mathbb{E}\left\{\left|h_{i i}(n)\right|^{2}\right\}}\right]^{+}
$$

where $[x]^{+}$is equivalent to $\max (0, x)$ and $\lambda_{i}$ is chosen to satisfy the power constraint (1) with equality.

Therefore, at a NE of game $\mathcal{G}$, each transmitter allocates its power by waterfilling on every subcarrier according to the information about the direct channel and interference statistics that its corresponding receiver has fed back after $L$ OFDM symbols have been observed.

At this point arises the questions of uniqueness of the NE in our game and whether or not it can be reached iteratively, which is the subject of the next section.

\section{B. Uniqueness and convergence conditions}

Sufficient conditions on equilibrium uniqueness and convergence of iterative waterfilling algorithms have been given in [7], [8] in the case of known channel realizations. These conditions are obtained by proving that utility functions are diagonally strictly concave, which implies NE uniqueness [18]. Starting from the expression (5), we can follow the same developments to find sufficient conditions on channel statistics guaranteeing NE uniqueness in the game $\mathcal{G}$. Let $\mathbf{Q} \in \mathbb{R}^{M \times M}$ be defined as

$$
[\mathbf{Q}(n)]_{i j}= \begin{cases}\frac{\mathbb{E}\left\{\left|h_{j i}(n)\right|^{2}\right\}}{g_{i}(n) \mathbb{E}\left\{\left|h_{i i}(n)\right|^{2}\right\}} & \text { if } i \neq j \\ 0 & \text { otherwise }\end{cases}
$$

The sufficient condition on NE uniqueness is then obtained by adapting [7, Theorem 2] to our context.

Theorem 1: Game $\mathcal{G}$ admits a unique NE if

$$
\rho(\mathbf{Q}(n))<1, \quad \forall n \in\{1, \cdots, N\},
$$

where $\mathbf{Q}(n)$ is defined in (14) and $\rho(\mathbf{Q}(n))$ is its spectral radius.

Convergence of iterative best-response algorithms is not guaranteed, except for some particular types of games [16]. 
In general, only sufficient conditions can be given and their proofs are application specific. We focus here on sequential waterfilling, for which conditions on convergence have been given in [8, Theorem 1]. Let $\mathbf{Q}^{\max } \in \mathbb{R}^{N \times N}$ be defined as

$$
\left[\mathbf{Q}^{\max }\right]_{i j}=\left\{\begin{aligned}
\max _{n} \frac{\mathbb{E}\left\{\left|h_{j i}(n)\right|^{2}\right\}}{g_{i}(n) \mathbb{E}\left\{\left|h_{i i}(n)\right|^{2}\right\}} & \text { if } i \neq j \\
0 & \text { otherwise }
\end{aligned}\right.
$$

Theorem 2: If the following condition is satisfied

$$
\rho\left(\mathbf{Q}^{\max }\right)<1
$$

then the sequential waterfilling algorithm converges to the unique NE of game $\mathcal{G}$.

These two conditions can be physically interpreted according to the average interference level perceived by each link. When the interference is high, multiple NE may exist and users will try to reach one of them by iteratively best-responding to each other. In this case, as the interferences become higher, the optimal strategies will tend towards FDMA-like power allocations where all the links communicate using orthogonal frequency bands [7], [15]. As shown in [7], [8] by simulations, the sufficient conditions for NE uniqueness and iterative waterfilling convergence are met when all the links are far enough from each other. However, having (C2) not satisfied does not imply that the algorithm cannot converge. As the simulations will show, iterative power adaption of the UA links according to the waterfilling policy can be highly beneficial even if both (C1) and (C2) hold false.

\section{Simulation Results}

We focus on two OFDM UA links with $N=256$ subcarriers in the same bandwidth $B=6 \mathrm{kHz}$ centered around $f_{c}=12$ $\mathrm{kHz}$. The subcarrier spacing is $\Delta f=23.4 \mathrm{~Hz}$. Direct and cross channels $h_{j i}, i, j \in\{1,2\}$ are generated by an UA shallow water channel simulator described in [19]. The powerdelay profiles are obtained by a ray tracing model driven by geometric parameters such as the transmitter/receiver range and their depths, and time fluctuations are modeled according to entropy maximization of the Doppler spectrum given the mean Doppler spread and the Rice factor of the main arrival. Path losses and frequency-dependent absorption are also taken into account using Thorp's formula [20].

We consider a shallow water communication scenario in a water depth of $50 \mathrm{~m}$ where the distance separating transmitters and receivers is $1 \mathrm{~km}$ for both direct and interference links. The depth of each terminal is arbitrarily chosen between 5 and $20 \mathrm{~m}$. A Doppler spread of $1 \mathrm{~Hz}$ and a maximum Rice factor of $10 \mathrm{~dB}$ are chosen to model the channel temporal fluctuations. The resulting frequency responses are depicted in Figure 1. A 20 second period is chosen for the power allocation update, which is long enough compared to the channel coherence time and the OFDM symbol duration to assume ergodicity. Thus, this corresponds also to the duration over which channels and interference statistics are computed by each receiver and to the feedback link period. The SNR is fixed to $15 \mathrm{~dB}$ relatively to the user experiencing the smallest attenuation. The noise PSD is modeled with a decay of $18 \mathrm{~dB} /$ decade. The total power constraint (1) is $P_{i}^{\max }=N$ for each transmitter.
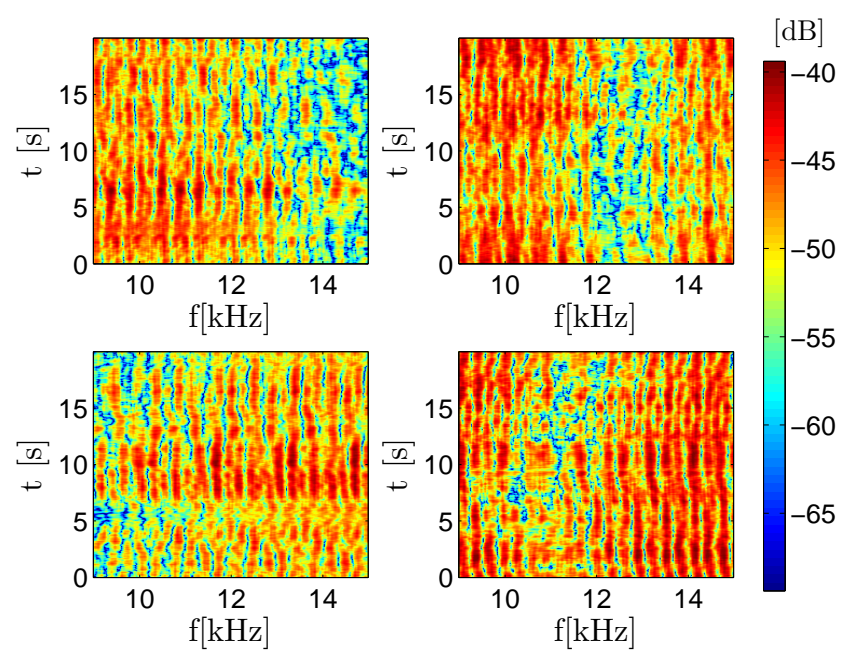

Fig. 1. Example of time-varying frequency responses of the directs and interference channels obtained from the simulator described in [19]. From top-left corner, clockwise : $h_{11}(t, f), h_{12}(t, f), h_{22}(t, f), h_{21}(t, f)$.

The initial PSD of both links is uniform and they update their strategy one after the other ${ }^{3}$ according to the waterfilling policy in (13). The game is played over 50 iterations. The evolution of players utility functions and their last power allocation are depicted in Figures 2 and 3. The latter is in agreement with the channel frequency responses of Figure 1. User 1 does not allocate its power on the last subcarriers since it experiences both attenuation and interferences from his opponent, whereas user 2 has more incentive to use frequencies in the 9 to $11 \mathrm{kHz}$ and 13 to $15 \mathrm{kHz}$ bands. In this scenario the sufficient conditions $(\mathrm{C} 1)$ and $(\mathrm{C} 2)$ hold false. However, one can see that more than $95 \%$ of the final utilities are reached before the $10^{t h}$ iteration, which corresponds to less than 3 minutes for a 20 second update period. At the last iterations of the game, the two players have reached a point where none of them has an interest to deviate. This point is one of the multiple NE of the scenario, and depending on the intial

\footnotetext{
${ }^{3}$ It is not necessary to assume some form of coordination since we can consider systems having the same update period but starting their transmissions at different times.
}

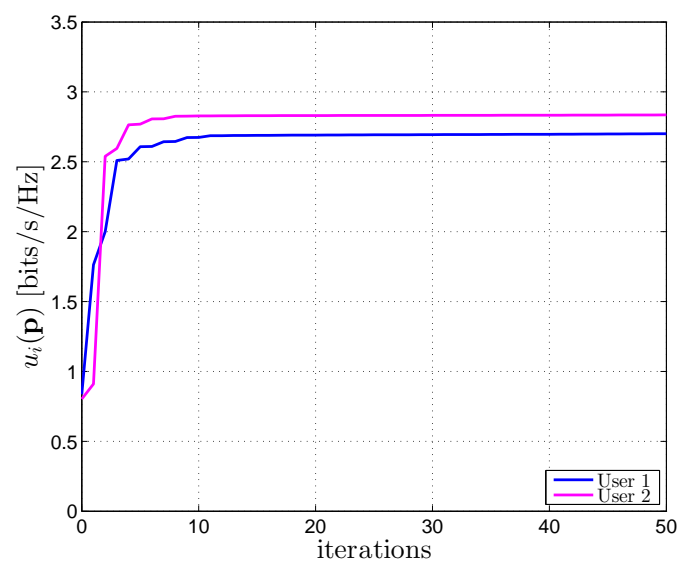

Fig. 2. Spectral efficiency of the players - (C1) and (C2) not satisfied. 


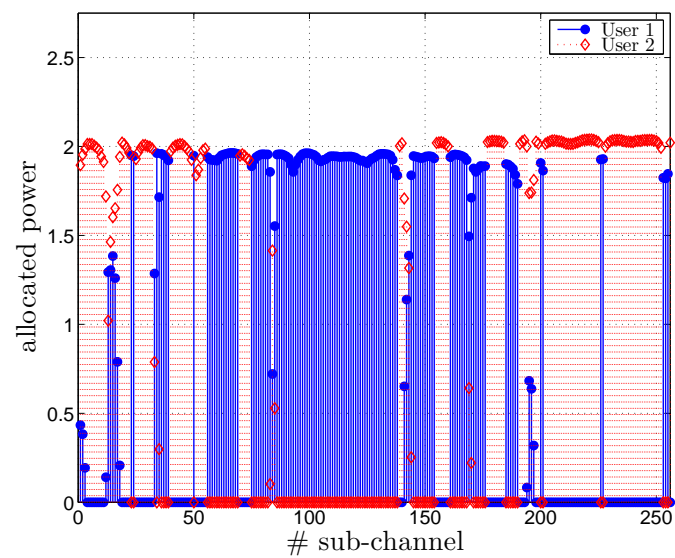

Fig. 3. Distributed power allocation after 50 iterations.

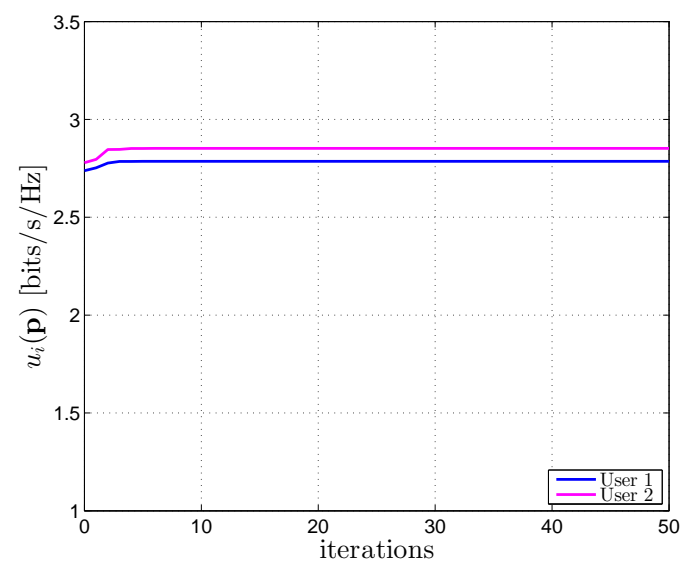

Fig. 4. Spectral efficiency of the players - (C1) and (C2) satisfied.

power allocation and who plays first, the players could have reached a slightly different one. All the simulations we have run on this scenario have shown this convergence behavior. Most importantly, the information rates of both players are almost tripled compared to the intial state.

To conclude, we consider a scenario of lower interference in order to make (C1) and (C2) hold true. We keep the same parameters, except the distance between transmitter $j$ and receiver $i \neq j$ are set to $3 \mathrm{~km}$ for both players. The resulting utilities are shown in Figure 4, where we can see only a small gain compared to the initial uniform power allocation. As the players become sufficiently far apart from each other, they are less prone to take the multiuser interferences into account so that their power allocation strategies tend to be waterfilling according only to their own channels and the noise.

\section{CONCLUSION}

A decentralized power control method for multiuser UA systems using OFDM has been studied. Based on game-theoretic tools, the proposed adaptive, distributed power allocation scheme allows UA links sharing the same physical resource to maximize their information rates in a noncooperative manner. The random time-varying nature of the UA channel as well as constraints posed by the low speed of sound on the feedback links have been taken into account. Simulation have shown that even with a few knowledge about the multiuser environment, UA links could almost triple their spectral efficiency in a high inteference setup. Future works will consider the impact of estimation errors on channels and interference statistics, as well as slow variations on the channels statistics, and provide results on real UA channels probed at sea.

\section{REFERENCES}

[1] E. McCarthy, International Regulation Of Underwater Sound: Establishing Rules and Standards to Address Ocean Noise Pollution, Kluwer Academic Publishers, 2004.

[2] P. van Walree, "Propagation and Scattering Effects in Underwater Acoustic Communication Channels," IEEE J. Ocean. Eng., vol. 38, no. 4, pp. 614-631, October 2013.

[3] P. Qarabaqi and M. Stojanovic, "Adaptive Power Control for Underwater Acoustic Communications," in Proc. of IEEE OCEANS 2011, June 2011.

[4] A. Radosevic, R. Ahmed, T.M. Duman, J. G. Proakis, and M. Stojanovic, "Adaptive OFDM Modulation for Underwater Acoustic Communications: Design Considerations and Experimental Results," IEEE J. Ocean. Eng., vol. 39, no. 2, pp. 357-370, Apr 2014.

[5] R. Otnes, A. Asterjadhi, P. Casari, M. Goetz, T. Husoy, I. Nissen, K. Rimstad, P. van Walree, and M. Zorzi, Underwater Acoustic Networking Techniques, Springer Briefs in Electrical and Computer Engineering, 2012.

[6] W. Yu, G. Ginis, and J. M. Cioffi, "Distributed Multiuser Power Control for Digital Subscriber Lines," IEEE J. Sel. Areas on Comm., vol. 20, no. 5, pp. 1105-1115, June 2002.

[7] G. Scutari, D. P. Palomar, and S. Barbarossa, "Optimal Linear Precoding Strategies for Wideband Non-Cooperative Systems Based on Game Theory-Part I: Nash Equilibria," IEEE Trans. Signal Processing, vol. 56, no. 3, pp. 1230-1249, March 2008.

[8] G. Scutari, D. P. Palomar, and S. Barbarossa, "Optimal Linear Precoding Strategies for Wideband Non-Cooperative Systems Based on Game Theory-Part II: Algorithms," IEEE Trans. Signal Processing, vol. 56, no. 3, pp. 1250-1267, March 2008.

[9] G. Scutari, D.P. Palomar, and S. Barbarossa, "Distributed totally asynchronous iterative waterfilling for wideband interference channel with time/frequency offset," in IEEE Int. Conf. Acoustics, Speech and Signal Processing (ICASSP) 2007, April 2007, vol. 4, pp. IV-1325-IV1328.

[10] R. Otnes, P. van Walree, and T. Jenserud, "Validation of Replay-Based Underwater Acoustic Communication Channel Simulation," IEEE J. Ocean. Eng., vol. 38, no. 4, pp. 689-700, 2013.

[11] F.-X. Socheleau, C. Laot, and J.-M. Passerieux, "Stochastic Replay of non-WSSUS Underwater Acoustic Communication Channels Recorded at Sea," IEEE Trans. Signal Process., vol. 59, no. 10, pp. 4838-4849, 2011.

[12] F-X. Socheleau, C. Laot, and J-M. Passerieux, "Parametric ReplayBased Simulation of Underwater Acoustic Communication Channels," IEEE J. of Oceanic Eng., vol. 40, no. 4, pp. 4838-4839, 2015.

[13] O. Oyman, H. Nabar, H. Bolcskei, and A.J. Paulraj, "Tight Lower Bound on the Ergodic Capacity of Rayleigh Fading MIMO Channels," IEEE Global Telecommunications Conference, 2002 (GLOBECOM'02), vol. 2, pp. 1172-1176, November 2002.

[14] F-X. Socheleau, A. Pottier, and C. Laot, "Stochastic Replay of SIMO Underwater Acoustic Communication Channels," OCEANS 2015, pp. 1-6, October 2015.

[15] A. Lesherm and E. Zehavi, "Game Theory and the Frequency Selective Interference Channel," IEEE Signal Processing Mag., vol. 26, pp. 28-40, September 2009.

[16] G. Bacci, S. Lasaulce, W. Saad, and L. Sanguinetti, "Game Theory for Networks: A tutorial on game-theoretic tools for emerging signal processing applications," IEEE Signal Processing Magazine, vol. 33, no. 1, pp. 94-119, January 2016.

[17] D. Fudenberg and J. Tirole, Game Theory, Cambridge MA MIT Press, 1991.

[18] J. B. Rosen, "Existence and Uniqueness of Equilibrium Points for Concave N-Persons Games," Econometrica, vol. 33, no. 3, pp. 521534, July 1965.

[19] F-X. Socheleau, C. Laot, and J-M. Passerieux, "Concise Derivation of Scattering Function from Channel Entropy Maximization," IEEE Trans. on Communications, vol. 58, no. 11, pp. 3098-3103, November 2010.

[20] R. J. Urick, Principles of Underwater Sound, 3rd Edition, McGraw-Hill, 1983. 